\title{
USO DE OLEATO DE ETANOLAMINA PARA HEMANGIOMAS DA CAVIDADE BUCAL: UM ESTUDO DE CINCO ANOS.
}

\section{USE OF ETHANOLAMINE OLEATE FOR HEMANGIOMA IN THE ORAL CAVITY: A STUDY OF FIVE YEARS}

\author{
Bruno Nifossi Prado* \\ Aline Franco dos Santos ${ }^{* *}$ \\ Juliane Mayara Magalhães Oliveira** \\ Gabriela Furst Vaccarezza*** \\ Claudio Fróes de Freitas ${ }^{* * * *}$
}

\begin{abstract}
RESUMO
Introdução: O hemangioma é uma lesão vascular, podendo representar uma neoplasia benigna dos vasos sanguíneos, um hamartoma ou uma malformação vascular (Neville et al. 1, 1998, Marcucci 2, 2005). O hemangioma representa $8 \%$ das lesões da cavidade oral do ambulatório da Universidade Cidade de São Paulo. (Prado et al. ${ }^{3}$, 2010). Métodos: foram avaliados 178 prontuários da Clínica de Odontologia da Universidade Cidade de São Paulo no período de 2005 a 2010. Na análise avaliou-se o gênero acometido, a faixa etária, a localização e tamanho da lesão, e a quantidade de aplicações de oleato de etanolamina para regressão total da lesão Resultados: O gênero masculino foi o mais acometido com $54 \%$ dos casos, a $6^{\text {a }}$ e a $7^{\mathrm{a}}$ décadas foram as mais prevalentes com uma média de 62 anos, o lábio inferior foi a região anatômica mais acometida e em $38 \%$ dos casos apenas uma aplicação foi necessária para a regressão total da lesão.
\end{abstract}

DESCRITORES: Epidemiologia • Hemangioma • Medicina bucal • Doenças da boca

\section{ABS TRACT}

Introduction: The hemangioma is a vascular lesion, which may represent a benign tumor of blood vessels, a hamartoma or a vascular malformation (Neville et al. ${ }^{1}$, 1998, Marcucci ${ }^{2}$, 2005). Hemangioma accounts for $8 \%$ of lesions of the oral cavity (Prado et al. ${ }^{3}, 2010$ ). Methods: We evaluated 178 medical records of clinical dentistry at the University City of São Paulo in the period 2005 to 2010 . The analysis assessed the affected gender, age, location and size of the lesion, and the number of applications of ethanolamine oleate to total regression of the lesion Results: Male gender was the most affected with 54\% of cases, the 6th and 7th decade were most prevalent with a mean of 62 years, the lower lip was the anatomic region most affected, an application was necessary in $38 \%$ of cases for regression of the lesion.

DESCRIPTORS: Epidemiology • Hemangioma • Oral medicine • Mouth diseases

\footnotetext{
* Cirurgião Buco-Maxilo-Facial do Hospital Vitória - São Paulo - Brasil. E-mail: brunoprado8@gmail.com

** Aluna de gradução em Odontologida da Universidade Cidade de São Paulo (UNICID).

*** Mestre em Ciências Odontológicas pela FO-USP. Professora de Odontologia e Medicina da Universidade Cidade de São Paulo (UNICID).

**** Diretor do curso de Odontologia da Universidade Cidade de São Paulo (UNICID).
} 


\section{N T RO DUÇÃ O}

O hemangioma é uma lesão vascular, podendo representar uma neoplasia benigna dos vasos sanguíneos, um hamartoma ou uma malformação vascular (Neville et al. ${ }^{1}, 1998$, Marcucci ${ }^{2}$, 2005). O hemangioma representa $8 \%$ das lesões da cavidade oral atendidas no ambulatório da Universidade Cidade de São Paulo (Prado et al. $\left.{ }^{3}, 2010\right)$.

Sua característica clínica pode ser uma bolha ou uma mancha de conteúdo sanguinolento, avermelhada ou azulada que desaparece momentaneamente à compressão digital ou vitropressão, retornando ao seu volume primitivo, podendo-se assim, estabelecer o diagnóstico clínico. (Boraks ${ }^{4}, 2001$, Toledo et al. $\left.{ }^{5}, 2004\right)$. São pulsáteis e têm temperatura mais elevada que os tecidos adjacentes, seu tamanho é variável indo de poucos milímetros até vários centímetros, podendo até causar uma assimetria facial (Boraks ${ }^{4}, 2001$ ).

Essas proliferações de vasos podem ser herdadas (genéticas), sendo comumente detectadas na infância ou ao nascimento, ou adquiridas, decorrentes de traumas, tendendo, assim, a serem assintomáticos (Neville et al. ${ }^{1}, 1998$ ).

O tratamento dos hemangiomas pode ser realizado de diversas maneiras, dependendo de sua extensão e localicalização (Neville et al. ${ }^{1}$, 1998) (Boraks ${ }^{4}, 2001$ ). Para as lesões menores e periféricas podese optar por esclerose química, excisão cirúrgica convencional, laserterapia, radioterapia, eletrocoagulação e crioterapia (Seo et al. ${ }^{6}, 2009$ ). Nas lesões maiores e/ ou intraósseas, o tratamento deve ser através de embolização ou obliteração da lesão e dos vasos adjacentes, se necessário (Assis et al. ${ }^{7}, 2009$, Loureiro et al. ${ }^{8}, 2010$ ).

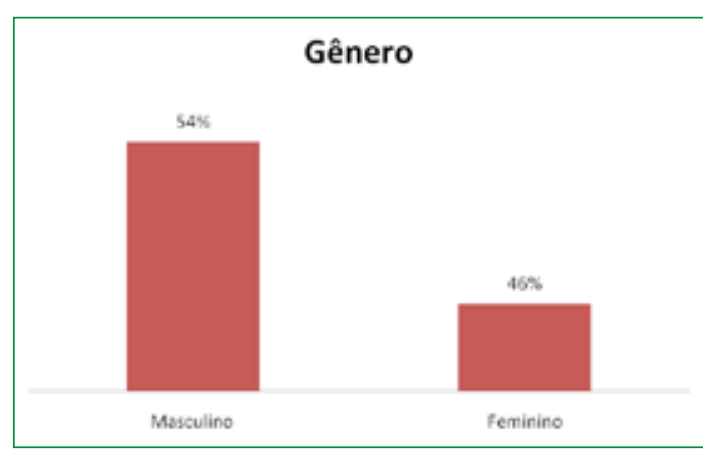

Figura 1
ISSN 1983-5183

A esclerose química com Oleato de Etanolamina apresenta excelentes resultados em hemangiomas da cavidade oral, podendo necessitar de mais de uma aplicação para regressão total do tumor. (Seo et al. ${ }^{6}, 2009$, Ribas et al. ${ }^{9}, 2004$, Johann et al. $\left.{ }^{10}, 2005\right)$.

O objetivo deste trabalho é apresentar um estudo retrospectivo de cinco anos em pacientes diagnosticados com hemangiomas na cavidade oral tratados com oleato de etanonolamina.

\section{MÉTODOS}

Para realização do estudo epidemiológico do tratamento de hemangiomas com oleato de etanolamina foram avaliados 178 prontuários da Clínica de Odontologia da Universidade Cidade de São Paulo no período de 2005 a 2010.

Todos os prontuários foram padronizados segundo Boraks ${ }^{4}$ (2001) e aqueles com o diagnóstico de hemangioma foram selecionados para obtenção dos dados. Os critérios de exclusão envolveriam preenchimento correto dos prontuários com identificação, diagnóstico, tratamento e evolução.

Na análise avaliou-se o gênero acometido, a faixa etária, a localização da lesão, e a quantidade de aplicações de oleato de etanolamina para regressão total da lesão.

Todos os dados tabulados, gráficos e tabelas foram desenvolvidos no programa Microsoft Excel 2007®.

\section{RESULTADOS}

Dos 178 prontuários avaliados na clínica odontológica durante cinco anos, 13 prontuários foram diagnosticados como hemangiomas da cavidade oral e tratados com aplicações de oleato de etanolamina.

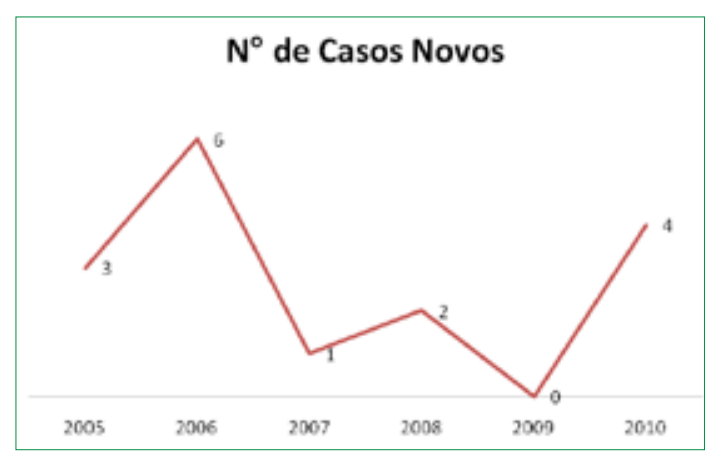

Figura 2
PRADO BN

SANTOS AF

OLIVEIRA JMM

VAcCAREzZa GF

FREITAS C F

USO DE OLEATO

DE ETANOLAMINA

PARA HEMANGI-

OMAS DA CAVI-

DADE BUCAL: UM

ESTUDO DE CINCO ANOS. $\therefore 43$

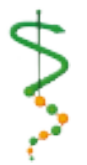

REVISTA DE ODONTOLOGIA DA UNIVERSI DADE CIDADE DE SÃO PAULO

$2011 ; 23(1): 42-5$ JAN - ABR 


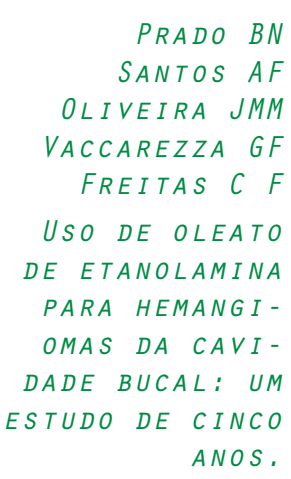

. $44 \ldots$

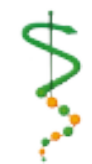

REVISTA DE

ODONTOLOGIA DA

UNIVERSIDADE

CIDADE DE SÃO

PAULO

$2011 ; 23(1): 42-5$,

$J A N-A B R$

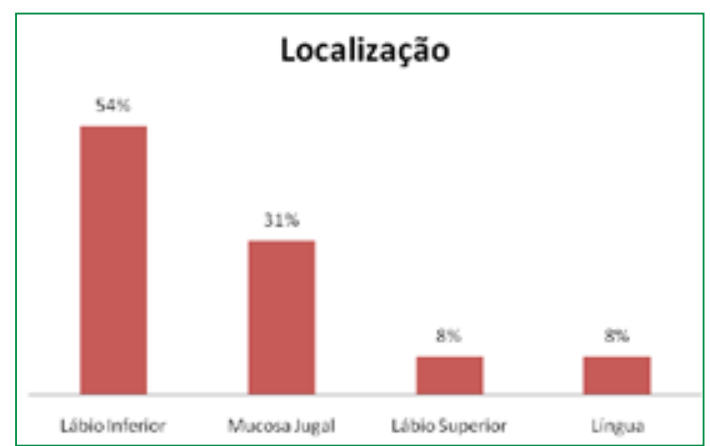

Figura 3

Em nosso estudo, os pacientes do gênero masculino com hemangiomas foram os mais prevalentes e apresentaram 7 casos, enquanto o gênero o feminino, 6 casos (Figura 1). A faixa etária mais prevalente foi entre a $6^{a}$ e $7^{a}$ décadas de vida, sendo que o paciente mais novo possui 34 anos de idade e o mais velho 76 , evidenciando uma idade média de 62 anos.

O número de novos casos apresentou seu pico de 6 casos em 2006 com decréscimo nos anos seguintes e um novo pico de crescimento no ano de 2010 (Figura 2).

A localização dos hemangiomas na cavidade oral teve o lábio inferior como região anatômica mais prevalente com 7 casos (54\%), seguido da mucosa jugal, 4 casos $(31 \%)$ e do lábio superior e língua ambos com 1 caso (8\%)( Figura 3). Os tamanhos dos hemangiomas foram variados o menor encontrado foi de $2 \mathrm{~mm}$ e o maior de $20 \mathrm{~mm}$, produzindo uma média de $6,8 \mathrm{~mm}$.

Para a regressão completa do hemangioma apenas 1 aplicação prevaleceu com 5 casos (38\%), seguida de 3 aplicações com 4 casos $(31 \%), 2$ aplicações com 3 casos (23\%) e 4 ou mais aplicações apenas com 1 caso relatado (8\%) (Figura 4).

\section{I SCUSSÃO}

O aparecimento de hemangiomas pode ser atribuído a uma alteração vascular hereditária diagnosticada na infância ou em pacientes adultos ou estar intimamente ligados ao trauma (Angelo et al. ${ }^{11}$, 2008). Os hemangiomas são lesões que dificilmente reapareceram ou sofrem malignização (Ribas et al. ${ }^{9}$, 2004).

Quanto ao gênero masculino foi o mais prevalente com 54\%, concordando com (Angelo et al. ${ }^{11}$ (2008) e discordando de

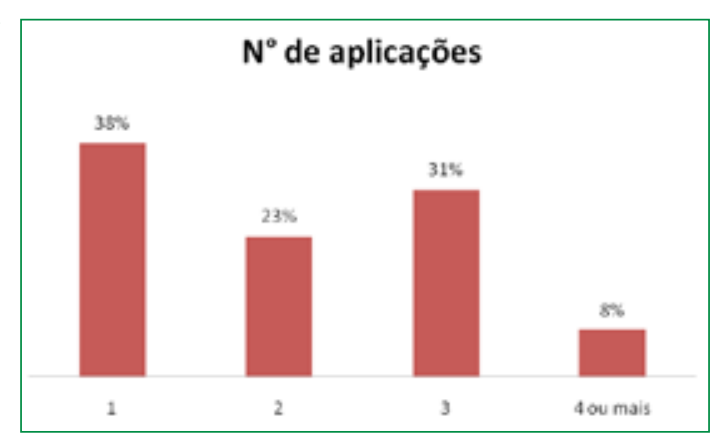

Figura 4

Johann et al. ${ }^{10}$ (2005) que apresentou o gênero feminino com 59\%. A idade mais acometida em nosso estudo foi entre a $6^{a}$ e $7^{a}$ décadas de vida, discordando de Angelo et al. ${ }^{11}$ (2008), com a faixa etária predominante de adolescentes de 13 a 18 anos. A região anatômica mais acometida foi o lábio inferior concordando com o estudo de Johann et al. ${ }^{10}$ (2005) e Angelo et al. ${ }^{11}$ (2008). A faixa etária mais incidente também pode ser atribuída às particularidades dos serviços e não estar associada a maior prevalência na população.

O tratamento para hemangiomas é controverso e depende da experiência e disponibilidade de recursos. A embolização torna-se essencial em hemangiomas intraósseos e hemangiomas de grandes dimensões onde há má formação, são provenientes de vasos de grande calibre (Loureiro et al. ${ }^{8}$, 2010). A ressecção cirúrgica é o tratamento com menos chances de recidiva e deve estar associado a eletrocoagulação na maioria das vezes (Toledo et al. ${ }^{5}$, 2004, Assis et al. ${ }^{7}, 2009$ ). A crioterapia é um método pouco utilizado em hemangiomas por ser muito doloroso e resultar em atrofia cutânea ou cicatriz (Seo et al. ${ }^{6}$, 2009). A escleroterapia é o método mais seguro tornando possível a regressão total ou parcial da lesão, facilitando a ressecção posteriormente (Seo et al. ${ }^{6}, 2009$ ).

Todos os nossos casos de hemangioma tratados com oleato de etanolamina obtiveram resultados favoráveis com regressão total da má formação. Johann et al. ${ }^{10}$ (2005) obteveram sucesso em 27 casos de hemangiomas tratados com oleato de etanolamina nas concentrações de 1,25\% e 2,5\%, não obtendo nenhuma significância estatística entre os dois tipos de concentração da substância injetada. Os hemangiomas tratados em nosso estudo por 
oleato de etanolamina não ultrapassaram o tamanho de $20 \mathrm{~mm}$ e o uso de apenas 1 aplicação prevaleceu em 38\% dos casos, obtendo-se uma média geral de 2 aplicações por paciente. No estudo elaborado por Johann et al. ${ }^{10}$ (2005) em hemangiomas com até $20 \mathrm{~mm}$, a média de aplicações foi de 1,9 para as concentrações de $1,25 \%$ de oleato de etanolamina e 2,2 para as concentrações de $2,25 \%$.

\section{CONCLUSÃO}

- Em nosso estudo de pacientes diag-
ISSN 1983-5183

nosticados e tratados com oleato de etanolamina, o gênero masculino foi o mais prevalente, a idade média foi de 62 anos, a localização anatômica mais acometida foi o lábio inferior, o maior hemangioma tratado foi de $20 \mathrm{~mm}$ e apenas uma aplicação foi necessária em 38\% dos casos.

- O tratamento com oleato de etanolamina apresentou $100 \%$ de sucesso nos pacientes com hemangiomas da cavidade oral.

\section{REFERÊNCIAS}

1. Neville B, Damm D, Allen C, Bouquot J. Patologia oral \& maxilofacial. Rio de Janeiro: Guanabara Koogan; 1998. 705p.

2. Marcucci G. Fundamentos da odontologia: estomatologia. Rio de Janeiro: Guanabara Koogan; 2005. 264p.

3. Prado B, Trevisan S, Passarelli D. Estudo epidemiológico das lesões bucais no período de 5 anos. Rev Odontol UNICID 2010 jan-abr; 22(1):25.

4. Boraks S. Diagnóstico bucal. São Paulo: Artes Médicas; 2001.

5. Toledo H, Castro E, Castro A, Soubhia A, FB SJ. Hemangioma cavernoso de lábio inferior: caso clínico. Revista Odontológica de Araçatuba 2004 jan-jun;25(1):9.

6. Seo J, Utumi E, Zambon C, Pedron I, Rocha A. Escleroterapia de hemangioma labial. Rev Odonto 2009 jul-dez; 17(34):106.

7. Assis G, Silva S, Moraes P, Amaral J, Germano A. Hemangioma de língua: relato de caso. Rev Cir Traumatol Buco-Maxilo-fac 2009 abr.-jun; 9(2):59.

8. Loureiro CC, Falchet PC, Gavranich J, Jr., Lobo Leandro LF. Embolization as the treatment for a life-threatening mandibular arteriovenous malformation. J Craniofac Surg 2010 Mar;21(2):380-2.

9. Ribas M, Laranjeira J, Sousa M. Hemangioma bucal: escleroterapia com oleato de etanolamina. Revisão da literatura e apresentação de caso. Rev de Clin Pesq Odontol 2004 out-dez;1(2):31.

10. Johann AC, Aguiar MC, do Carmo MA, Gomez RS, Castro WH, Mesquita RA. Sclerotherapy of benign oral vascular lesion with ethanolamine oleate: an open clinical trial with 30 lesions. Oral Surg Oral Med Oral Pathol Oral Radiol Endod 2005 Nov;100(5):579-84.

11. Angelo A, Moraes J, Rosa M, Duarte R, DeBiase R. Incidência de hemangioma na região de cabeça e pescoço em pacientes com a faixa etária entre 18 anos: estudo de 10 anos. Rev Odontol UNICID 2008 mai.-ago;20(2):209.

Recebido em: 23/03/2011

Aceito em: 28/03/2011 